J. Korean Math. Soc. 49 (2012), No. 5, pp. 893-905

http://dx.doi.org/10.4134/JKMS.2012.49.5.893

\title{
A FAST NUMERICAL METHOD FOR SOLVING A REGULARIZED PROBLEM ASSOCIATED WITH OBSTACLE PROBLEMS
}

\author{
DaMing Yuan, Xi Li, and ChengFeng Lei
}

\begin{abstract}
Kirsi Majava and Xue-Cheng Tai [12] proposed a modified level set method for solving a free boundary problem associated with unilateral obstacle problems. The proximal bundle method and gradient method were applied to solve the nonsmooth minimization problems and the regularized problem, respectively. In this paper, we extend this approach to solve the bilateral obstacle problems and employ Rung-Kutta method to solve the initial value problem derived from the regularized problem. Numerical experiments are presented to verify the efficiency of the methods.
\end{abstract}

\section{Introduction}

The obstacle problem is a typical example of the elliptic variational inequality of the first kind. Many important problems, such as the torsion of an elasticplastic cylinder, the Stefan problem can be formulated by transformation to an obstacle problem [15]. Several comprehensive monographs can be consulted for the theory and numerical solution of variational inequalities $[4,5,23]$. Since obstacle problem is highly nonlinear, it is difficult for the computation of approximate solutions. So far, the problem is usually solved by projection method [5], active set strategy [9], multigrid and multilevel method [6, 10, 11, 23], level set method [12], piecewise linear system method [2], discontinuous Galerkin method [21], etc.

In this paper, we extend the modified level set method, which for solving the unilateral obstacle problem [12], to solve the bilateral obstacle problem. To minimize the total energy $\mathcal{J}(u)$, we introduce the time variable and then apply the Rung-Kutta method to solve an initial value problem of an ordinary differential equation derived from the regularized problem.

Received June 13, 2010; Revised December 27, 2011.

2010 Mathematics Subject Classification. 65K10, 90C33.

Key words and phrases. Rung-Kutta method, level set method, obstacle problem.

(C)2012 The Korean Mathematical Society 


\subsection{Mathematical formulation of the unilateral obstacle problem}

Suppose $\Omega \subset \mathbb{R}^{2}$ is a bounded Lipschitz domain, with boundary $\partial \Omega$. $\varphi \in$ $H^{1}(\Omega) \cap C(\bar{\Omega})$ is a given function satisfying $\varphi \leq 0$ on $\partial \Omega, f \in L^{2}(\Omega)$ is a given function representing external force for physical problems. The unilateral obstacle problem describes the equilibrium position $u$ of an elastic membrane constrained to lie above the given obstacle $\varphi$ under the action of the force $f$. From the Lagrange principle of minimizing the total energy, the obstacle problem can be posed as a problem in the calculus of variations, i.e., find $u \in \mathcal{K}_{1}$ such that

$$
\mathcal{J}(u)=\min _{v \in \mathcal{K}_{1}} \mathcal{J}(v)
$$

where

$$
\begin{gathered}
\mathcal{J}(v)=\frac{1}{2} \int_{\Omega}|\nabla v|^{2} d x d y-\int_{\Omega} f v d x d y, \\
\mathcal{K}_{1}=\left\{v \in H_{0}^{1}(\Omega), \varphi \leq v \text { a.e. in } \Omega\right\} .
\end{gathered}
$$

As $\mathcal{K}_{1}$ is a convex set, the minimization problem (1)-(3) is equivalent to a first kind of elliptic variational inequality [5], i.e., find $u \in \mathcal{K}_{1}$ such that

$$
\int_{\Omega} \nabla u \cdot \nabla(v-u) d x d y \geq \int_{\Omega} f(v-u) d x d y, \quad \forall v \in \mathcal{K}_{1} .
$$

To find the solution of the unilateral obstacle problem, one needs to find the contact region. We denote the region $\Omega^{*}=\{x \mid u(x)=\varphi(x), x \in \Omega\}$ and its boundary $\partial \Omega^{*}$. If the solution $u \in C^{2}(\Omega) \cap H_{0}^{1}(\Omega)$, the variational problem (1)-(3) or the variational inequality (4) is equivalent to the following partial differential system:

$$
\left\{\begin{array}{l}
-\triangle u-f=0 \text { and } u>\varphi \text { if } x \in \Omega \backslash\left(\Omega^{*} \cup \partial \Omega^{*}\right), \\
-\triangle u-f \geq 0 \text { and } u=\varphi \text { if } x \in\left(\Omega^{*} \cup \partial \Omega^{*}\right), \\
u=0 \text { if } x \in \partial \Omega,
\end{array}\right.
$$

where $\triangle$ is the Laplace operator. Once the contact region $\Omega^{*}$ is found, the problem becomes a boundary value problem with the Possion equation outside $\Omega^{*}$ and the solution $u$ equals to the obstacle function in $\Omega^{*}$ as well as on its boundary $\partial \Omega^{*}$. Since the region $\Omega^{*}$ is unknown prior, the problem is a free boundary problems essentially.

\subsection{Mathematical formulation of the bilateral obstacle problem}

As for the bilateral obstacle problem, it can also be posed as a problem in the calculus of variations in the form of (1)-(3) or a first kind elliptic variational inequality in the form of (4) but the convex set

$$
\mathcal{K}_{2}=\left\{v \in H_{0}^{1}(\Omega), \varphi \leq v \leq \psi, \text { a.e. in } \Omega\right\} .
$$

Where $\varphi, \psi \in H^{1}(\Omega) \cap C(\bar{\Omega})$ are functions satisfying $\varphi \leq \psi$ in $\Omega$ and $\varphi \leq 0, \psi \geq$ 0 on $\partial \Omega$. We denote the two contact regions $\Omega^{-}=\{x \mid u(x)=\varphi(x), x \in \Omega\}$, 
$\Omega^{+}=\{x \mid u(x)=\psi(x), x \in \Omega\}$ and their boundaries $\partial \Omega^{-}, \partial \Omega^{+}$and their closure $\overline{\Omega^{-}}, \overline{\Omega^{+}}$, respectively. Similarly, the corresponding piecewise can be formulate as

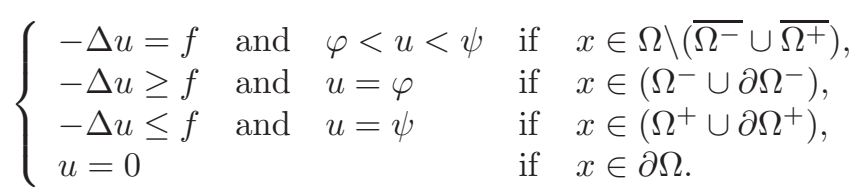

For the bilateral obstacle problems, there are two contact regions $\Omega^{-}$and $\Omega^{+}$. Once the regions $\Omega^{-}, \Omega^{+}$are found, the problems become a boundary value problem with the Possion equation outside the regions $\Omega^{-}, \Omega^{+}$and the solution $u$ equals $\varphi$ in $\Omega^{-} \cup \partial \Omega^{-}$and $\psi$ in $\Omega^{+} \cup \partial \Omega^{+}$.

\section{A modified level set method for solving unilateral obstacle problems}

Level set method is a numerical technique which offers highly robust and accurate methods for tracking the evolution of interfaces. One can find details about the theory, implementation and application of the methods in $[13,14$, $16,19]$.

Level set method consists of defining the unknown boundary $\Gamma$ of a region in the Euclidean space $\mathbb{R}^{2}$ as the zero level set of a family of level set functions $\left\{\phi(x, t), x \in \mathbb{R}^{2}, t \geq 0\right\}$, i.e.,

$$
\Gamma(t)=\{x \mid \phi(x, t)=0\}
$$

with $\Gamma(0)$ being the initial curve and $\Gamma(t)$ converging to the true free boundary when $t \rightarrow \infty$. Assuming the free boundary propagates along its normal direction with speed $F$, one can obtain the Hamilton-Jacobian equation as [14]

$$
\begin{gathered}
\phi_{t}+F|\nabla \phi|=0, \\
\phi(x, 0)=\phi_{0}(x) .
\end{gathered}
$$

Where $\phi_{0}= \pm$ distance $(x, \Gamma(0))$, i.e., a signed distance function.

In standard level set method, the interface $\Gamma$ is moved by updating the corresponding level set function through (8), (9).

In [12], the authors proposed an alternative approach for the level set idea and used it to trace the free boundary problem which arises from the unilateral obstacle problem. More precisely, after defining the Heaviside function $H(\phi)$ as

$$
H(\phi)= \begin{cases}1 & \phi>0 \\ 0 & \phi \leq 0,\end{cases}
$$

then for any $v \in \mathcal{K}_{1}$, there exists a $\phi \in H_{0}^{1}(\Omega)$ such that

$$
v(\phi)=\varphi+\phi H(\phi) .
$$


When smoothing the Heaviside function $H(\phi)$ by the approximation of

$$
H_{\epsilon}(\phi)=\frac{1}{\pi} \tan ^{-1} \frac{\phi}{\epsilon}+\frac{1}{2}
$$

one can turn the minimization problem (1) into an unconstrained minimization problem

$$
\min _{\phi \in H_{0}^{1}(\Omega)} \mathcal{J}^{\epsilon}(\phi)
$$

where $\epsilon$ is a parameter and $\mathcal{J}^{\epsilon}(\phi)$ is a differentiable cost functional obtained from $\mathcal{J}(u)$ after substituting $u$ by $u(\phi)=\varphi+\phi H_{\epsilon}(\phi)$. Then a gradient method with the step size $\alpha$ can be employed to update the function $\phi$ in the form of

$$
\phi^{n+1}=\phi^{n}-\alpha \frac{\partial \mathcal{J}^{\epsilon}}{\partial \phi}\left(\phi^{n}\right)
$$

with the stopping criterion $\left\|\frac{\partial \mathcal{J}^{\epsilon}}{\partial \phi}\left(\phi^{n}\right)\right\|<$ tolerance.

Remark 1. From (11), it is easy to see that $v(\phi)=\varphi$ if $\phi \leq 0$ (i.e., $x \in$ $\Omega^{*} \cup \partial \Omega^{*}$ ) and $v(\phi)=\varphi+\phi$ if $\phi>0$ (i.e., $x \in \Omega \backslash \overline{\Omega^{*}}$ ). So in the modified level method, the level set function $\phi$ can be used to represent the interface $\partial \Omega^{*}$ and carry information about the solution $u$ outside or inside the contact region $\Omega^{*}$. However, in the standard method the level set function $\phi$ is used to represent the interface alone. Beside (11), another form of smooth approximation of $u(\phi)=\varphi+\frac{1}{2}\left(\varphi+\sqrt{\phi^{2}+\epsilon}\right)$ is also considered in [12].

Remark 2. The standard level set method relies on two central embeddings, i.e., the embedding of the interface as the zero level set of a higher dimensional function and the embedding of the interface's velocity to this higher dimensional level set function. How to extent the velocity of the interface $\Gamma_{0}$ to the whole domain $\Omega$ and construct the signed distance function are two critical techniques when applying the method. These are usually not easy to carry out, one can see $[14,1,17,18]$. However, when applying the modified level set method, one can use iterative type of method to find the value of level set function $\phi$. In this sense, the modified method is simpler than the standard one.

\section{Solving the bilateral obstacle problem}

\subsection{The modified level set method for the bilateral obstacle problem}

In this section, we extend the idea of the modified level set method to track the two free boundary curves $\partial \Omega^{-}$and $\partial \Omega^{+}$in the bilateral obstacle problem.

For any $v \in \mathcal{K}_{2}$, there exists $\phi \in H_{0}^{1}(\Omega)$ such that

$$
v=\varphi+\phi(\psi-\varphi)[H(\phi)-H(\phi-1)]+(\psi-\varphi) H(\phi-1) .
$$


It is easy to see that

$$
v=\left\{\begin{array}{lll}
\varphi & \text { if } \phi \leq 0 & \text { (i.e., in the lower contact region), } \\
\phi(\psi-\varphi)+\varphi & \text { if } 0<\phi \leq 1 & \text { (i.e., outside the contact region), } \\
\psi & \text { if } \phi>1 & \text { (i.e., in the upper contact region) }
\end{array}\right.
$$

From these, we can see that the level set function $\phi$ carries the information of the solution $v \in \mathcal{K}_{2}$, i.e., lies inside the contact regions $\Omega^{-}, \Omega^{+}$or outside these regions, i.e., lies inside $\Omega \backslash\left(\Omega^{-} \cup \Omega^{+}\right)$.

Denotes $\delta_{\epsilon}(\phi)$ the gradient of $H_{\epsilon}(\phi)=\frac{1}{\pi} \tan ^{-1} \frac{\phi}{\epsilon}+\frac{1}{2}$, then it is easy to obtain

$$
\delta_{\epsilon}(\phi)=\frac{\epsilon}{\pi\left(\phi^{2}+\epsilon^{2}\right)}
$$

and the partial derivative

$$
\frac{\partial v}{\partial \phi}=(\psi-\varphi)\left[H_{\epsilon}(\phi)-H_{\epsilon}(\phi-1)+\frac{\epsilon\left(\epsilon^{2}+\phi(1-\phi)\right)}{\pi\left(\phi^{2}+\epsilon^{2}\right)\left((\phi-1)^{2}+\epsilon^{2}\right)}\right]
$$

after replacing the Heaviside function $H(\phi)$ by $H_{\epsilon}(\phi)$.

From (2), the derivative of the cost functional $\mathcal{J}^{\epsilon}(v)$ can consequently be expressed as

$$
\begin{aligned}
\frac{\partial \mathcal{J}^{\epsilon}}{\partial \phi} & =\frac{\partial \mathcal{J}^{\epsilon}}{\partial v} \frac{\partial v}{\partial \phi} \\
& =(-\Delta v-f)(\psi-\varphi)\left[H_{\epsilon}(\phi)-H_{\epsilon}(\phi-1)+\frac{\epsilon\left(\epsilon^{2}+\phi(1-\phi)\right)}{\pi\left(\phi^{2}+\epsilon^{2}\right)\left((\phi-1)^{2}+\epsilon^{2}\right)}\right] .
\end{aligned}
$$

We can solve the regularized minimization problem (12) associated with the bilateral obstacle problem by the gradient method analogously. In this paper, we will propose a fast numerical method to find the level set function $\phi$ in the following section. As for the other form of smooth approximation $u(\phi)=\varphi+\frac{1}{2}\left(\varphi+\sqrt{\phi^{2}+\epsilon}\right)$, a similar process can be carried out, but these will not be further pursued in this paper except for numerical tests.

\subsection{Rung-Kutta method for solving the regularized minimization problem}

After introducing the time variable $t$ as a parameter and denote the level set function $\phi$ as $\phi(t)$, we can easily derive that

$$
\frac{d \mathcal{J}^{\epsilon}}{d t}=\frac{\partial \mathcal{J}^{\epsilon}}{\partial v} \frac{\partial v}{\partial \phi} \frac{d \phi}{d t}
$$

Different from the standard level set method or the gradient method [12], we update the level set function $\phi(t)$ by solving an initial value problem

$$
\left\{\begin{array}{l}
\frac{d \phi}{d t}=m(t) F(t, \phi) \\
\phi(0)=\phi_{0}
\end{array}\right.
$$


where $F(t, \phi)$ is defined as $-\frac{\partial \mathcal{J}^{\epsilon}}{\partial v} \frac{\partial v}{\partial \phi}(0 \leq t<+\infty)$ and $m(t)>0$ which usually is taken as a constant and $\phi_{0}$ is the initial guess of $\phi$. It is easy to see that $\frac{d \mathcal{J}^{\epsilon}}{d t} \leq 0$ according to the definition of $F(t, \phi)$ and (18), so the cost functional $\mathcal{J}^{\epsilon}$ decreases with the time $t$.

It is well known that Rung-Kutta methods are important to find the numerical solution of an ordinary differential equation. In the fast numerical method, which we propose in this paper, the standard fourth-order Runge-Kutta method form is employed to solve the problem (19).

\section{Numerical results}

We present the results of numerical experiments on the proposed algorithms. All experiments are performed on Dell workstation (3.00GHZ Pentium(R)4 CPU) with MATLAB codes.

In the following examples, the domains $\Omega$ are divided with $\bar{n}$ subintervals in $x$-direction and $y$-direction. Denote $n=\bar{n}^{2}$ and $h$ the spatial step. Then $u, f, \phi, \Phi$ and $\psi$ are vectors in $\mathbb{R}^{n}$, whose components correspond to the values of the functions in the equidistant discretization points in the domain $\Omega$. A five point finite difference approximation is used for $-\Delta$ with Dirichlet boundary conditions. $e\left(u^{*}, \bar{u}\right)$ denotes the average error between the analytical solution $\bar{u}$ and the obtained results $u^{*}$ :

$$
e\left(u^{*}, \bar{u}\right)=\sqrt{\frac{1}{n} \sum_{i=1}^{n}\left(u^{*}-\bar{u}\right)_{i}^{2}}
$$

In the case of a unilateral obstacle, we denote $F\left(u^{*}\right)$ the value of the cost functional for the computed result. We consider two approaches of smoothed approximation of the Heaviside function and denote $H_{\varepsilon}(\Phi)=\frac{1}{\pi} \arctan \frac{\Phi}{\varepsilon}+\frac{1}{2}$ for Approach 1 and $H_{\varepsilon}(\Phi)=\sqrt{\Phi^{2}+\epsilon^{2}}$ for Approach 2. The stopping criterion is $\left\|\frac{d \mathcal{J}}{d t}\right\| \leq 10^{-4}$ which is equivalent to the criterion used in the [12] and $m(t)=1$.

\subsection{Unilateral obstacle problems}

In this section, we compare the efficiency between our method and the method in [12]. The same example has been considered, so is the discretization of the domain and Laplace operator.

Example 1. In this example, we choose $\Omega=(-2,2) \times(-2,2)$ and the upper obstacle function

$$
\phi(x, y)=\left\{\begin{array}{lll}
\sqrt{1-x^{2}-y^{2}} & \text { for } \quad x^{2}+y^{2} \leq 1 \\
-1, & \text { elsewhere }
\end{array}\right.
$$

With the consistent Dirichlet boundary condition, problem (1) with the obstacle function $\phi$ in (21) has an analytical solution of the form

$$
v(x, y)= \begin{cases}\sqrt{1-x^{2}-y^{2}} & \text { for } r \leq r^{*}, \\ -r^{* 2} \ln (r / R) / \sqrt{1-r^{* 2}} & \text { for } r \geqslant r^{*}\end{cases}
$$


where $r=\sqrt{x^{2}+y^{2}}, R=2$ and $r^{*}=0.6979651482 \cdots$ which satisfies

$$
\left(r^{*}\right)^{2}\left(1-\ln \left(r^{*} / R\right)\right)=1 \text {. }
$$
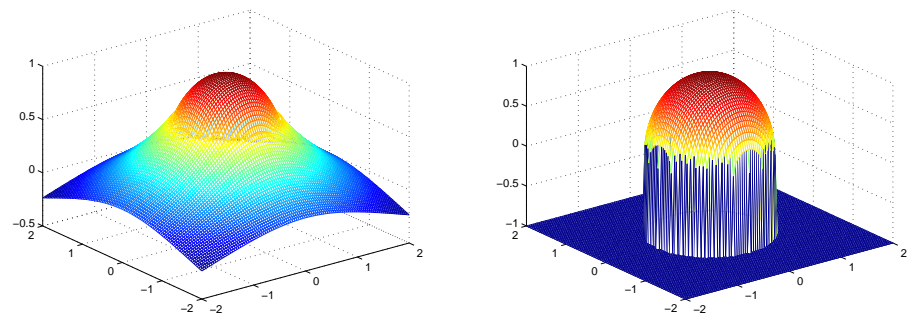

Figure 1. (a) The analytical solution and (b) The obstacle $(\bar{n}=128)$.

The obstacle and the analytical solution are illustrated in Figure 1. The same example has been considered in [12] as Example 4.2.

TABLE 1. The results in Example 1, where $\bar{n}=30$, the parameter $\epsilon=h^{2}$ and the initial guess of $\Phi_{0}=1$. PB and GR is the method used in [12] and RK is the method used in this paper.

\begin{tabular}{cllcl}
\hline Appr & Alg & it & $e\left(u^{*}, \bar{u}\right)$ & $\mathrm{F}\left(u^{*}\right)$ \\
\hline 1 & GR & 34991 & $3.54 \times 10^{-3}$ & 3.8728 \\
2 & & 200238 & $8.93 \times 10^{-4}$ & 3.9196 \\
\hline 1 & PB & 4742 & $3.55 \times 10^{-3}$ & 3.8728 \\
2 & & 48509 & $8.83 \times 10^{-4}$ & 3.9198 \\
\hline 1 & RK & 444 & $4.00 \times 10^{-3}$ & 3.8905 \\
2 & & 484 & $4.00 \times 10^{-3}$ & 3.9461 \\
\hline
\end{tabular}

4.1.1. Results of the experiments. In Table 1 , we take $\bar{n}=30$, the smoothing parameter $\epsilon=h^{2}$ and the time step $\Delta t=0.1$ in RK method. The first column indicates which smoothed approach is stated. In the next two column the solution algorithm used is given and it denotes the number of iterations needed. In the fourth column, $e\left(u^{*}, \bar{u}\right)$ denotes the average error between the analytical solution $\bar{u}$ and the obtained result $u^{*}$. In the last column, $F\left(u^{*}\right)$ denotes the value of the cost functional for the obtain result. From this table, we observe that the RK method is faster than PB and GR.

We consider the influence of the time step $\Delta t$ and the smoothed parameter $\epsilon$ on the error. In Table 2, the results are showed when we take the initial 
TABle 2. The results of Approach 1 and Approach 2 by the Rung-Kutta method in Example 1 for the initial guess $\Phi_{0}=$ $0.5, \bar{n}=30$ and the different smoothed parameters from $h^{2}-$ $h^{6}$. The time step $\Delta t=0.01$ are considered.

\begin{tabular}{cccccc}
\hline Time & $\epsilon$ & Appr & it & $e\left(u^{*}, \bar{u}\right)$ & $\mathrm{F}\left(u^{*}\right)$ \\
\hline 0.01 & 1 & 5859 & $1.17 \times 10^{-2}$ & 3.9082 \\
& 2 & 5844 & $1.42 \times 10^{-2}$ & 3.9611 \\
\hline & $h^{2}$ & 1 & 5861 & $9.90 \times 10^{-3}$ & 3.9478 \\
& 2 & 5854 & $1.03 \times 10^{-2}$ & 3.9545 \\
\hline & $h^{3}$ & 5 & 5865 & $9.70 \times 10^{-3}$ & 3.9532 \\
& & $h^{4}$ & 5863 & $9.70 \times 10^{-3}$ & 3.9540 \\
\hline & 1 & 5868 & $9.60 \times 10^{-3}$ & 3.9539 \\
& $h^{5}$ & 2 & 5866 & $9.70 \times 10^{-3}$ & 3.9540 \\
\hline & 1 & 6363 & $1.43 \times 10^{-2}$ & 3.9860 \\
& 2 & 5901 & $9.60 \times 10^{-3}$ & 3.9540 \\
\hline
\end{tabular}

guess of level set function $\Phi=0.5$ and $\epsilon=h^{2}, h^{3}, h^{4}, h^{5}, h^{6}$ and $\Delta t=0.01$ respectively.

When comparing the other parameters of $\Delta t$ and $\epsilon$, we find that there are appropriate smoothed parameters $\epsilon$ corresponding to the different time step $\Delta t$. For $\Delta t=0.5$ and the same parameter $\epsilon$, we can't obtain a convergence result. This is very similar to the CFL condition, which is necessary for the convergence when applying finite difference method to approximate the wave equation [3]. In this respect, it is different from the method in [12].
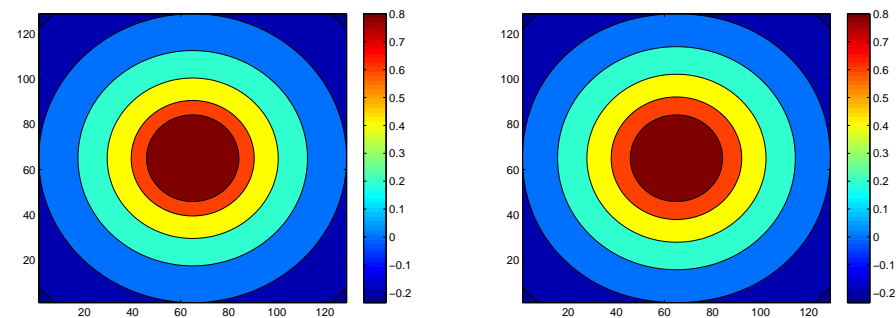

Figure 2. (a) The contour surface of the analytical solution for Example 1 and (b) The contour surface of the obtained solution for Example 1, where $\bar{n}=128, \epsilon=h^{3}, \Delta t=0.1$ and the initial guess of level set function $\Phi_{0}=1$.

In Figure 2, we compare the contour surf of the analytic solution and the computed one when $\bar{n}=128, \epsilon=h^{3}, \Delta t=0.1$ and the initial guess of level set function $\Phi_{0}=1$ 


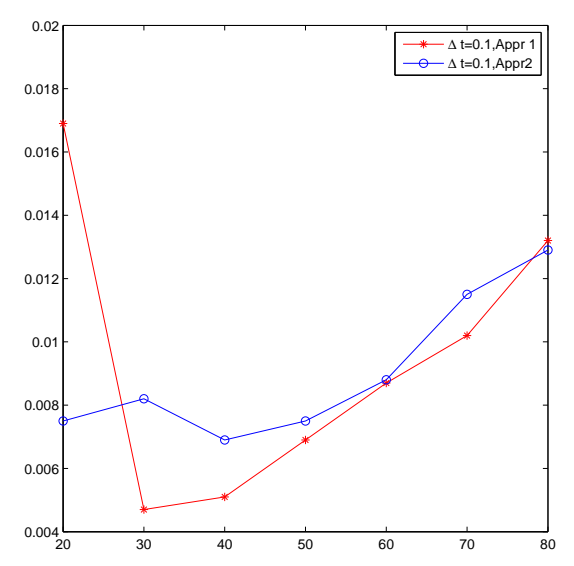

FIGURE 3. The errors relative to the different $\bar{n}$. Here we take $\bar{n}=20,30,40,50,60,70,80$, the time step $\Delta t=0.1, \Phi_{0}=1$ and the smoothed parameter $\epsilon=h^{3}$.

In Figure 3, we show the errors relative to the different $\bar{n}$. Here we take $\bar{n}=$ $20,30,40,50,60,70,80$, the time step $\Delta t=0.1$ and the smoothed parameter $\epsilon=h^{3}$. We find there exists a critical number $\bar{n}$ for which the error is optimal, and the same for other number $\bar{n}$ and the time step $\Delta t$.

\subsection{Bilateral obstacle problem}

Example 2. This example, which concern one-dimensional case, is designed to testify the effectiveness of the proposed algorithm. We choose the domain $\Omega=[0,2 \pi]$ and the upper obstacle function $\psi=\frac{\sqrt{3}}{3}$ in the interval $\left[\frac{\pi}{3}, \frac{2 \pi}{3}\right]$ and the lower obstacle function $\phi=-\frac{\sqrt{3}}{3}$ in the interval $\left[\frac{4 \pi}{3}, \frac{5 \pi}{3}\right]$. With the homogeneous Dirichlet boundary condition, the minimization problem (1) has an analytical solution of the form

$$
\bar{u}(x)=\left\{\begin{array}{cl}
\sin (x)-\frac{3}{2 \pi} x, & \text { if } 0 \leq x \leq \frac{\pi}{3} \\
\frac{\sqrt{3}}{3}, & \text { if } \frac{\pi}{3}<x \leq \frac{2 \pi}{3} \\
\sin (x)+\frac{3}{2 \pi} x-\frac{3}{2}, & \text { if } \frac{\pi}{3}<x \leq \frac{2 \pi}{3} \\
\frac{-\sqrt{3}}{3}, & \text { if } \frac{4 \pi}{3}<x \leq \frac{5 \pi}{3} \\
\sin (x)-\frac{3}{2 \pi} x+\sqrt{3}, & \text { if } \frac{5 \pi}{3}<x \leq 2 \pi .
\end{array}\right.
$$

The analytical solution and the approximation, which presented in blue solid line and red dash-dot line respectively, are showed in Figure 4. Under the error strategy $(20)$, we get the error $e\left(u^{*}, \bar{u}\right)=1.14 \times 10^{-2}$. In Figure 5 , the 


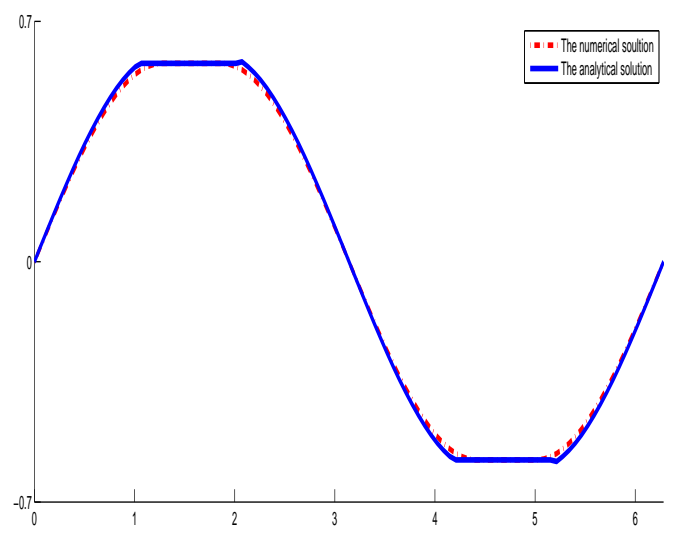

Figure 4. The exact solution (blue solid line) and the approximation (red dash-dot line) in Example 3.1 when $n=100$.

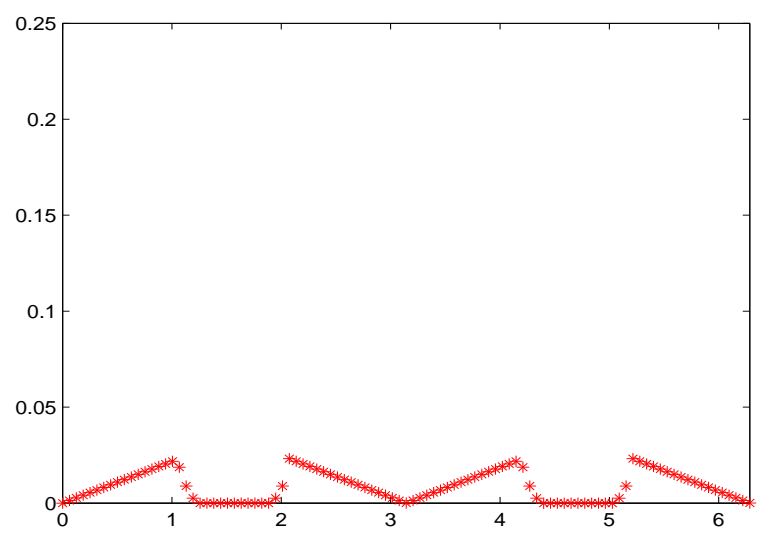

Figure 5. The pointwise absolute difference between the exact solution and the approximation.

pointwise absolute difference between the exact solution and the approximation are showed. Due to the singularity near the separatrices where the active set and inactive set are switched, one can see that the error is relative bigger therein. The influence can be reduced by the adaptive technique [7].

Example 3. As a test problem, we consider a two-dimension case which had been reported in $[20,9]$, i.e., suppose $\Omega=(0,1) \times(0,1)$. Let $\varphi(x, y)=$ 
$-\operatorname{dist}((x, y), \partial \Omega), \psi(x, y)=0.2$, and set

$$
f(x, y)
$$

$= \begin{cases}300, & \text { if }(x, y) \in S=\{(x, y) \in \Omega:|x-y| \leq 0.1 \text { and } x \leq 0.3\}, \\ -70 \exp (y) g(x), & \text { if } x \leq 1-y \text { and }(x, y) \notin S, \\ 15 \exp (y) g(x), & \text { if } x>1-y \text { and }(x, y) \notin S,\end{cases}$

where

$$
g(x)= \begin{cases}6 x, & \text { if } 0<x \leq 1 / 6, \\ 2(1-3 x), & \text { if } 1 / 6<x \leq 1 / 3, \\ 6(x-1 / 3), & \text { if } 1 / 3<x \leq 1 / 2, \\ 2(1-3(x-1 / 3)), & \text { if } 1 / 2<x \leq 2 / 3, \\ 6(x-2 / 3), & \text { if } 2 / 3<x \leq 5 / 6, \\ 2(1-3(x-2 / 3)), & \text { if } 5 / 6<x \leq 1\end{cases}
$$
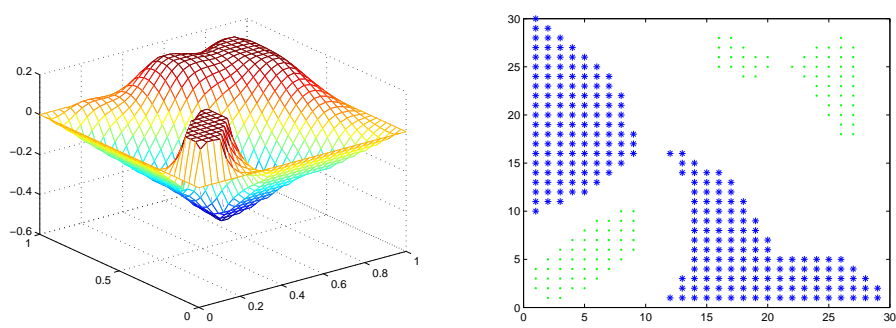

Figure 6. (a) The approximated solution and (b) The the lower $(*)$ and the upper $(\cdot)$ coincidence sets when $\bar{n}=30$, $\epsilon=h^{3}, \Phi_{0}=0$ in Example 3 .

When taking $\bar{n}=30, \epsilon=h^{3}$ and the level-set function $\Phi_{0}=0$, we apply the Approach 1 to this problem and show the approximated solution and coincidence sets in Figure 6.

\section{Conclusion}

A modified level-set method is proposed for solving the free boundary problems derived from the unilateral and bilateral obstacle problems. This method does not need to solve a Hamilton-Jacobi equation of a level-set function to trace the interface. When a time-dependent problem is taken as the "evolution" of the level-set function $\Phi$, we can employ the method of solving the ordinary differential equation, classically the fourth-order Rung-Kuatta method, to obtain the function at each iteration. Then we get a more effective method for solving the regularized problem. In numerical tests, we find the time step $\Delta t$, the initial guess of the level-set function $\Phi_{0}$, the smoothed parameter $\epsilon$ and the number of the subinterval $\bar{n}$ have influence on the error. There are critical values for minimal error. 
Acknowledgement. This work is partially supported by the Chinese National Science Foundation (No. 10871179) and Youth Fund of Department of Education of JiangXi Province (GJJ09492). The authors would like to thank Prof. Xiao-Liang Cheng (ZheJiang University, P. R. China) and Prof. W. Han (Iowa University, USA) for their helpful discussions.

\section{References}

[1] D. Adalsteinsson and J. A. Sethian, The fast construction of extension velocities in level set methods, J. Comput. Phys. 148 (1999), no. 1, 2-22.

[2] L. Brugnano and V. Casulli, Iterative solution of piecewise linear systems, SIAM J. Sci. Comput. 30 (2008), no. 1, 463-472.

[3] R. Courant, K. Friedrichs, and H. Lewy, On the partial difference equaton of mathematical physics, IBM J. Res. Dev. 11 (1928), no. 2, 215-234.

[4] G. Duvaut and J. L. Lions, Inequalities in Mechanics and Physics, Springer-Verlag, Berlin, Germany, 1976.

[5] R. Glowinski, Numerical Methods for Nonlinear Variational Problems, Springer, New York, 1984.

[6] R. Hoppe, Multigrid algorithms for variational inequalities, SIAM J. Numer. Anal. 24 (1987), 1046-1065.

[7] R. Hoppe and R. Kornhuber, Adaptive multilevel methods for obstacle problems, SIAM J. Numer. Anal. 31 (1994), no. 2, 301-323.

[8] S. Howison, F. Wilmott, and J. Dewynne, The Mathematics of Financial Derivative, Cambridge University Press, Cambridge, 1995.

[9] T. Kärkkäinen, K. Kunisch, and P. Tarvainen, Augmented Lagrangian active set methods for obstacle problems, J. Optim. Theory Appl. 119 (2003), no. 3, 499-533.

[10] R. Kornhuber, Monotone multigrid methods for elliptic variational inequalities I, Numer. Math. 69 (1994), no. 2, 167-184.

[11] _ Monotone multigrid methods for elliptic variational inequalities II, Numer. Math. 72 (1996), no. 4, 481-499.

[12] K. Majava and X.-C. Tai, A level set method for solving free boundary problems associated with obstacles, Int. J. Numer. Anal. Model. 1 (2004), no. 2, 157-171.

[13] S. Osher and R. Fedkiw, Level Set Method and Dynamic Implicit Surfaces, Springer, NewYork, 2000.

[14] S. Osher and J. A. Sethian, Fronts propagating with curvature dependent speed: Algorithms based on Hamilton-Jacobi formulation, J. Comput. Phys. 79 (1988), no. 1, $12-49$.

[15] J. Rodrigues, Obstacle Problems in Mathematical Physics, Elsevier Science 1987.

[16] J. A. Sethian, Level Set Methods, Evolving Interfaces in Geometry, Fluid Mechanics, Computer Vision and Material Science, Cambridge University Press, Cambridge, 1996.

[17] _ Numerical algorithms for propagating interfaces: Hamilton-Jacobi equations and conservation laws, J. Differential Geom. 31 (1990), no. 1, 131-161.

[18] _ A fast marching level set method for monotonically advancing fronts, Proc. Nat. Acad. Sci. U.S.A. 93 (1996), no. 4, 1591-1596.

[19] J. A. Sethian and J. D. Strain, Crystal growth and dendritic solidification, J. Comput. Phys. 98 (1992), no. 2, 231-253.

[20] F. Wang and X. L. Cheng, An algorithm for solving the double obstacle problems, Appl. Math. Comput. 201 (2008), no. 1-2, 221-228.

[21] F. Wang, W. M. Han, and X. L. Cheng, Discontinuous Galerkin methods for solving elliptic variational inequalities, SIAM J. Numer. Anal. 48 (2010), no. 2, 708-733.

[22] L. Xue and X. L. Cheng, An algorithm for solving the obstacle problems, Comput. Math. Appl. 48 (2004), no. 10-11, 1651-1657. 
A FAST NUMERICAL METHOD FOR SOLVING A REGULARIZED PROBLEM 905

[23] Y. Zhang, Multilevel projection algorithm for solving obstacle problems, Comput. Math. Appl. 41 (2001), no. 12, 1505-1513.

DAMing YuAN

College of Mathematics and Information Science

NANCHANG HANGKONG UNIVERSITY

NANChang 330063, P. R. China

AND

Department of Mathematics

ZHEJIANG UNIVERSITY

HANGZhou 310027, P. R. China

E-mail address: dmzju@126.com

XI LI

College of Mathematics and Information Science

NANChANG HANGKong University

NANChANG 330063, P. R. China

E-mail address: lixixi4321@126.com

ChengFeng Lei

College of Mathematics and Information Science

NANCHANG HANGKONG UNIVERSITY

NANChANG 330063, P. R. China

E-mail address: lcfxj@163.com 